\title{
Las vocaciones científicas en Educación Primaria. Una investigación inicial en Perú y España
}

Esther Cascarosa Salillas. Universidad de Zaragoza

Marta Marco. Colegio Santa Ana, Zaragoza

Recepción: 22.06.2018 | Aceptado: 15.09.2018

Correspondencia a través de ORCID: Esther Cascarosa

iD 0000-0002-3696-7673

Citar: Cascarosa, E. y Marco, M. (2018). Las vocaciones científicas en Educación Primaria. Una investigación inicial en Perú y España. ReiDoCrea, 7, 190-201.

Resumen: En este trabajo se presenta una investigación inicial que trata de discernir algunas de las razones del bajo número de vocaciones científicas entre los alumnos de dos países, Perú y España. Para ello, se analizan varios aspectos de la enseñanza de las ciencias en ambos países a través del análisis de aspectos tales como el currículo oficial, la dedicación horaria a la enseñanza de las ciencias en las clases de Educación Primaria o la formación en ciencias del profesorado. Los resultados obtenidos se completan con los de la observación directa en un centro de educación de cada uno de los dos países. Esto ha permitido sacar algunas conclusiones relacionadas con la importancia que se le da a la materia de ciencias dentro de las aulas de Primaria.

Palabras clave: Formación de docentes de primaria | Enseñanza de las ciencias

Scientific vocations in Elementary Education. An initial research in Peru and Spain

Abstract: This paper presents an initial research that tries to discern some of the reasons for the low number of scientific vocations among the students of two countries, Peru and Spain. To do this, various aspects of the teaching of science in both countries are analysed through the analysis of aspects such as the official curriculum, time dedication to the teaching of science in elementary education classes or training in Sciences of the Faculty. The results obtained are completed with the direct observation in a centre of education of each of the two countries. This has allowed some conclusions related to the importance given to the matter of science in the elementary classroom.

Keywords: Primary Teacher Education | Basic Science Education

\section{Introducción}

Para cualquier país, es fundamental que sus jóvenes desarrollen vocaciones científicas que culminen aportando saber y desarrollo a la sociedad. Estas vocaciones científicas deben desarrollarse desde los primeros años de la persona y, por lo tanto, el contexto educativo debe desempeñar una labor crucial. En este sentido, es bien conocido que se deben propiciar entornos de alfabetización científica que motiven al alumno y que lo inciten a descubrir las ciencias (Brenneman, 2011; Harlen, 2015).

Un aspecto que obstaculiza la inclinación de los estudiantes hacia las ciencias es la metodología que se utiliza en el proceso de enseñanza-aprendizaje. Es fundamental dejar atrás la rutina de cumplir con enseñar solo los aspectos teóricos de la ciencia y acercarla de manera práctica al aula. Las aulas deberían ser lugares en los que se propicie que el estudiante vaya más allá de la simple memorización de hechos, siendo capaces de tomar la iniciativa y ser responsables de su propio aprendizaje (Cuevas, Lee, Hart y Deaktor, 2005). También las investigaciones en didáctica de las ciencias concluyen con la necesidad de partir de la práctica como la prueba que posteriormente ayude a los alumnos a pensar e interpretar desde un marco teórico cada vez más abstracto, llegando a conclusiones a partir de dicha práctica (Izquierdo, Sanmartí y Espinet, 1999). Dicho proceso ayuda a trabajar diferentes procedimientos intelectuales que el docente deberá valorar y enseñar, siendo este un papel transcendental en las 
actividades prácticas. Pues, al contrario, si no se realiza de manera correcta, el pretendido aprendizaje a través de la práctica se convierte en el criticado aprendizaje autónomo, en el cual se espera que el niño observe la realidad y sea capaz de inferir todos los conocimientos científicos (García y Martínez, 2001).

Para que esto sea así, la labor del docente es fundamental. Respecto a esto es muy importante que los profesores estén bien formados en todas las áreas a impartir, pero en concreto en ciencias, y en este campo, tan importante es la formación en contenidos como en metodología, procedimientos y actitudes (Oliva y Acevedo, 2005). Una mala formación científica de los maestros desemboca en una baja confianza en sí mismo frente a la enseñanza de las ciencias propiciando así las clases magistrales sobre diferentes contenidos conceptuales que se deberían impartir por medio de propuestas prácticas (Cortes et. al, 2012). De esta forma, la formación del profesorado debería basarse en situaciones en las que tengan que poner en práctica tareas de indagación, para hacerlos conscientes de sus propias dificultades y aumentar la confianza en sí mismos, ya que, como señalan Liang y Richardson (2009), si en la formación inicial no se consigue que los futuros profesores se sientan confiados con esta forma de trabajar, se mantendrán poco familiarizados e incómodos cuando comiencen a desarrollar su labor profesional (Vílchez y Bravo, 2015).

En cuanto a la formación de los futuros maestros de Primaria, Pujol (2008) indica que el conocimiento científico del futuro profesorado de Educación Primaria se encuentra muy lejos de los mínimos deseables y presenta dificultades para abordar la Didáctica de las Ciencias. Este autor, además destaca la importancia de formar maestros en diferentes áreas: saber enseñar ciencias, saber gestionar grupos de escolares, saber participar en las dinámicas de los centros escolares, saber impulsar proyectos de innovación, saber posicionarse ante las nuevas situaciones emergentes y saber establecer interrelaciones con múltiples organizaciones y agentes externos a la escuela. A pesar de que las investigaciones sobre la educación científica concluyen con la importancia de formar a los futuros maestros no solo en contenidos, sino también en procedimientos, los futuros docentes en formación creen que lo más importante para saber dar clases de ciencias es conocer los aspectos teóricos (o disciplinares). Un $83 \%$ de los encuestados (futuros maestros de Educación Primaria) por Cortés et. al (2012) manifestaron que lo más importante es conocer la teoría que van a impartir y solo el $41 \%$ de los encuestados, le dio importancia a los aspectos metodológicos o didácticos.

Así, la renovación de la enseñanza de las ciencias no requiere únicamente la innovación de los materiales de aprendizaje y la superación de sus deficiencias, sino que debería haber una modificación en los planes de formación docente (Hodson, 1996), pues es imprescindible que el profesor sepa analizar los materiales y sea honesto a la hora de saber con qué objetivo se eligen las actividades para los alumnos (Nott, 1996). Esta formación debe partir de la propia reflexión del docente sobre su propia práctica educativa (Hewson y Hewson, 1987), siendo importante para los formadores conocer la realidad de las aulas y las concepciones, los conocimientos y las creencias de los alumnos a los que va dirigida.

Por último, no hay que olvidar que las políticas públicas educativas en ciencia desempeñan un papel que influye directamente en el desarrollo científico de los alumnos y finalmente del país, por lo tanto, es otro factor a contemplar.

Teniendo en cuenta todo lo anterior, en el presente trabajo se presenta una investigación inicial donde se analizan distintos aspectos de la enseñanza de las ciencias en dos países con un bajo número de vocaciones científicas, Perú y España. 


\section{Métodos}

Se han analizado varios aspectos de la enseñanza de las ciencias tanto en Perú como en España, donde se han tenido en cuenta las siguientes variables. Por un lado, se han analizado aspectos relacionados con las políticas educativas de ambos países y su repercusión sobre la evolución en la tasa de alfabetización. Sobre las ciencias en concreto, se ha estudiado el currículo oficial de ambos países en relación a los alumnos de Educación Primaria, la importancia de estas dentro de los currículos, las horas/semana que se trabajan las ciencias en ambos países y las competencias que se trabajan a través de las ciencias. Por último, se ha analizado la formación específica en ciencias del profesorado de Educación Primaria. Todo esto, se ha analizado a través de la lectura de documentos oficiales.

Por otro lado, y con el fin de estudiar in situ las metodologías aplicadas en el aula y analizar qué competencias se trabajan en la enseñanza de las ciencias, se han recogido datos basados en la observación directa en un aula de ciencias de cada país (Perú y España). En ambos casos, la observación se ha llevado a cabo con alumnos de la misma edad ( 7 años) en cursos equivalentes en uno y otro país $\left(2^{\circ} \mathrm{EP}\right)$. También el tiempo de observación ha sido el mismo (6 semanas).

De todo lo anterior se han obtenido los resultados que se muestran a continuación.

\section{Resultados}

\section{Políticas Educativas}

Para poder analizar las políticas educativas de ambos países con una visión amplia, parece conveniente estudiar algunos puntos previos que faciliten la comprensión del contexto de ambos como son: densidad de población, el gasto público en sanidad y en educación (ver Tabla 1).

Tabla 1: Aspectos generales de Perú y España (datos extraídos de www.datosmacro.com)

\begin{tabular}{|l|l|l|}
\hline & PERÚ & ESPAÑA \\
\hline Superficie & $1.285 .216,20 \mathrm{~km}^{2}$ & $505.370 \mathrm{~km}^{2}$ \\
\hline Población & 31.826 .018 habitantes & 46.549 .045 habitantes \\
\hline Densidad de población & 24,76 habitantes $/ \mathrm{km}^{2}$ & 92,11 habitantes $/ \mathrm{km}^{2}$ \\
\hline Gasto público en sanidad & $\begin{array}{l}15 \% \rightarrow 165 € / \text { habitante } \\
14,5 \% \rightarrow 1.521 € / \text { habitante }\end{array}$ \\
\hline Gasto público en educación & \begin{tabular}{l}
$\begin{array}{l}17,6 \% \rightarrow \\
€ / \text { habitante }\end{array}$ \\
\hline
\end{tabular} & $9,59 \% \rightarrow 973 € /$ habitante \\
\hline
\end{tabular}

Se observa que Perú tiene una superficie de territorio mucho mayor que España, y en función del número de habitantes de cada país, hace que su densidad de población sea menor de 25 habitantes $/ \mathrm{km}^{2}$. Esto significa que la población está repartida por todo el territorio, lo cual, en relación a los centros educativos, se traduce en que existen muchas escuelas rurales frente al número de escuelas en territorio urbano. En España, las escuelas se reparten también por el territorio, sin embargo, cada vez más escuelas rurales cierran debido a la falta de alumnado.

Por otra parte, se observa que la inversión de dinero por habitante tanto en sanidad como en educación son mayores en España que en Perú. En concreto, en educación se invierte casi cinco veces más euros/habitante en España. Esto debería repercutir 
directamente en el sistema educativo, ya sea en recursos materiales, infraestructuras o profesorado.

En la Tabla siguiente se muestra la evolución de las tasas de alfabetización en ambos países. Se puede observar como en los últimos 35 años la tasa de alfabetización en Perú ha mejorado notablemente, aumentando de 81,92\% de adultos alfabetos en 1981 hasta $94,17 \%$ en 2016 . Esto se ha debido en parte a la puesta en marcha de políticas educativas efectivas. También en España es patente el resultado de la inversión en educación realizada en los últimos años. En ambos países, en el año 2016 se describen tasas parecidas de alfabetización científica entre los jóvenes, siendo en Perú menor en las mujeres que en los hombres jóvenes.

Tabla 2: Evolución de la tasa de alfabetización según edad y sexo en porcentaje sobre la población total en Perú y en España (Datos de www.datosmacro.com).

\begin{tabular}{|c|c|c|c|c|c|c|c|c|}
\hline & Adultos & & $\begin{array}{l}\text { Jóvenes } \\
\text { Mujeres }\end{array}$ & & $\begin{array}{l}\text { Jóvenes } \\
\text { Hombres }\end{array}$ & & Jóvenes & \\
\hline Fecha & Perú & España & Perú & España & Perú & España & Perú & España \\
\hline 2016 & 94,17 & 98,25 & 98,67 & 99,61 & 99,09 & 99,64 & 98,88 & 99,62 \\
\hline 2012 & 89,59 & 97,89 & 98,68 & 99,71 & 98,72 & 99,64 & 98,70 & 9,67 \\
\hline 2007 & 89,59 & 97,94 & 96,71 & 99,56 & 98,01 & 99,58 & 97,36 & 99,57 \\
\hline 2004 & 87,67 & 97,17 & 95,66 & 99,53 & 97,80 & 99,58 & 96,76 & 99,56 \\
\hline 1991 & 87,15 & 96,49 & 93,79 & 99,57 & 96,99 & 99,54 & 95,37 & 99,55 \\
\hline 1981 & 81,92 & 92,81 & 89,76 & 98,64 & 96,51 & 98,64 & 93,12 & 98,64 \\
\hline
\end{tabular}

A pesar de esta importante evolución, actualmente en Perú hay de un millón de personas analfabetas. El $75 \%$ de ellas son adultos mayores (más de 65 años) y el resto son jóvenes con edades entre los 15 y 20 años. Según cifras del INEI (Instituto Nacional de Estadística e Informática), las 5 regiones con mayor porcentaje de analfabetismo son: Huánuco con $16,6 \%$ de analfabetos, Huancavelica con 14,9\%, Apurímac con 14,5\%, Ayacucho $13,8 \%$ y Cajamarca $11,8 \%$. Las regiones con menores tasas de analfabetismo son Lima $(2,3 \%)$, el Callao $(2,3 \%)$, Ica $(3,1 \%)$, Tumbes $(3,4 \%)$ y Arequipa $(4,2 \%)$ Todas por debajo del promedio nacional.

\section{Currículo y distribución horaria}

En relación al currículo se han recopilado las asignaturas impartidas en cada país, así como las horas asignadas a cada una de esas asignaturas. Estos resultados se muestran en la Tabla 3.

Tabla 3: Organización y distribución del tiempo semanal (en minutos) de Educación Primaria en Perú y en España.

\begin{tabular}{|l|c|c|c|c|c|c|c|}
\hline EN PERÚ & \multicolumn{10}{l|}{} \\
\hline ÁREAS & \multicolumn{7}{l|}{ GRADOS DE ESTUDIO } \\
\cline { 2 - 8 } CURRICULARES & $1^{\circ}$ & $2^{\circ}$ & $3^{\circ}$ & $4^{\circ}$ & $5^{\circ}$ & $6^{\circ}$ & TOTAL \\
\hline Matemática & 300 & 300 & 240 & 240 & 240 & 240 & 1.560 \\
\hline Comunicación & 300 & 300 & 240 & 240 & 240 & 240 & 1.560 \\
\hline Inglés & 120 & 120 & 180 & 180 & 180 & 180 & 960 \\
\hline Personal Social & 180 & 180 & 240 & 240 & 240 & 240 & 1.320 \\
\hline Arte y Cultura & 180 & 180 & 180 & 180 & 180 & 180 & 1.080 \\
\hline Ciencia y Tecnologia & 180 & 180 & 240 & 240 & 240 & 240 & 1.320 \\
\hline Educación física & 180 & 180 & 180 & 180 & 180 & 180 & 1.080 \\
\hline Educación religiosa & 60 & 60 & 60 & 60 & 60 & 60 & 360 \\
\hline Tutoría y Orientación & 120 & 120 & 120 & 120 & 120 & 120 & 720 \\
\hline
\end{tabular}




\begin{tabular}{|c|c|c|c|c|c|c|c|}
\hline \multicolumn{8}{|l|}{ educativa } \\
\hline $\begin{array}{l}\text { Horas de libre } \\
\text { disponibilidad }\end{array}$ & 180 & 180 & 120 & 120 & 120 & 120 & 840 \\
\hline TOTAL DE HORAS & 1.800 & 1.800 & 1.800 & 1.800 & 1.800 & 1.800 & 10.800 \\
\hline \multicolumn{8}{|l|}{ EN ESPAÑA } \\
\hline AREAS & \multicolumn{7}{|c|}{ GRADOS DE ESTUDIO } \\
\hline CURRICULARES & $1^{\circ}$ & $2^{\circ}$ & $3^{\circ}$ & $4^{\circ}$ & $5^{\circ}$ & $6^{\circ}$ & TOTAL \\
\hline Matemáticas & 225 & 225 & 225 & 225 & 225 & 225 & 1.350 \\
\hline $\begin{array}{l}\text { Lengua castellana y } \\
\text { literatura }\end{array}$ & 225 & 225 & 225 & 225 & 225 & 225 & 1.350 \\
\hline $\begin{array}{l}\text { Ciencias de la } \\
\text { naturaleza }\end{array}$ & 90 & 90 & 90 & 90 & 90 & 90 & 540 \\
\hline Ciencias sociales & 90 & 90 & 90 & 90 & 90 & 90 & 540 \\
\hline Lengua extranjera & 150 & 150 & 150 & 150 & 150 & 150 & 900 \\
\hline Educación física & 180 & 180 & 180 & 180 & 135 & 135 & 990 \\
\hline $\begin{array}{l}\text { Religión / Valores } \\
\text { sociales y cívicos }\end{array}$ & 45 & 45 & 45 & 45 & 45 & 45 & 270 \\
\hline Educación artística & 120 & 120 & 120 & 120 & 120 & 120 & 720 \\
\hline Tutoría & 45 & 45 & 45 & 45 & 45 & 45 & 270 \\
\hline $\begin{array}{l}\text { OTRAS } \\
\text { ACTIVIDADES } \\
\text { LECTIVAS }\end{array}$ & $1^{\circ}$ & $2^{\circ}$ & $3^{\circ}$ & $4^{\circ}$ & $5^{\circ}$ & $6^{\circ}$ & \\
\hline $\begin{array}{l}\text { Lenguas propias de } \\
\text { Aragón }\end{array}$ & 90 & 90 & 90 & 90 & 90 & 90 & 540 \\
\hline $\begin{array}{l}\text { Segunda lengua } \\
\text { extranjera }\end{array}$ & & & & & 90 & 90 & 180 \\
\hline Autonomia de centro & 180 & 180 & 180 & 180 & 225 & 225 & 1.170 \\
\hline TOTAL DE HORAS & 1.350 & 1.350 & 1.350 & 1.350 & 1.350 & 1.350 & 8.100 \\
\hline
\end{tabular}

De acuerdo a estos datos, se observan diferencias entre ambos países. En primer lugar, en Perú se dedican 450 horas al año más a ir a la escuela que en España. En cuanto al tiempo destinado a las ciencias, en Perú la dedicación horaria a ciencias evoluciona con la edad del alumnado. Los dos primeros años de Educación Primaria se destinan 180 horas/semana a trabajar las ciencias y pasan a ser 240 a partir del tercer curso. Cabe destacar que las ciencias se trabajan conjuntamente con la tecnología. No es así en España, que son una materia independiente y donde la dedicación horaria es constante (90 horas) a lo largo de la etapa de Primaria. Se observa también que en ambos países hay varias materias, encabezando esta lista Matemáticas y Comunicación (Lengua y Literatura en España) que tienen una dedicación horaria semanal muy superior a la enseñanza de las ciencias.

En España, las competencias en educación son responsabilidad de las Comunidades Autónomas. Los contenidos de ciencias se trabajan con el fin de conseguir alcanzar unas competencias. De las siete que generales que se deben trabajar en Educación Primaria, las que se trabajan específicamente desde el área de ciencias son: la competencia en el conocimiento y la interacción con el mundo físico, la competencia social y ciudadana, el sentido de iniciativa y espíritu emprendedor y la competencia lingüística.

Por otra parte, en Perú, el currículo oficial es competencia del Estado. En el desarrollo de dicho currículo se traban 29 competencias, de las cuales las que se asocian a ciencias son: la indagación mediante métodos científicos para construir sus conocimientos, explicación del mundo físico basándose en conocimientos sobre los seres vivos; materia y energía; biodiversidad, Tierra y Universo. 
En ambos países, la asignatura de ciencias se supera si se alcanzan las competencias estipuladas en el currículo. A modo de ejemplo, en la Tabla 4 se presentan los niveles de desarrollo de la competencia "indagación mediante métodos científicos para construir sus conocimientos", necesarios para considerar que se ha adquirido dicha competencia en los distintos cursos de Educación Primaria en Perú.

Tabla 4: Descripción del nivel requerido de la competencia "Indaga mediante métodos científicos para construir sus conocimientos" en cada nivel educativo en Perú.

\begin{tabular}{|c|c|c|}
\hline Nivel 8 & $\begin{array}{l}\text { Nivel } \\
\text { destacado }\end{array}$ & $\begin{array}{l}\text { Indaga a partir de preguntas sobre una situación y } \\
\text { argumenta la influencia de las variables, formula una o más } \\
\text { hipótesis con base a conocimientos científicos y } \\
\text { observaciones previas. Elabora el plan de indagación con } \\
\text { base en principios científicos y los objetivos planteados. } \\
\text { Realiza mediciones y comparaciones sistemáticas que } \\
\text { evidencian el comportamiento de las variables. Analiza } \\
\text { tendencias y relaciones en los datos tomando en cuenta la } \\
\text { teoría de errores, reproducibilidad y representatividad de la } \\
\text { muestra, los interpreta con principios científicos y formula } \\
\text { conclusiones. Evalúa la fiabilidad de los métodos y las } \\
\text { interpretaciones. Argumenta sus conclusiones basado en } \\
\text { sus resultados y conocimiento científico. A partir de sus } \\
\text { resultados formula nuevos cuestionamientos y evalúa el } \\
\text { grado de satisfacción que da la respuesta a la pregunta de } \\
\text { indagación. }\end{array}$ \\
\hline Nivel 7 & $\begin{array}{l}\text { Nivel } \\
\text { esperado } \\
\text { al final del } \\
\text { ciclo VII }\end{array}$ & $\begin{array}{l}\text { Indaga a partir de preguntas y plantea hipótesis con base en } \\
\text { conocimientos científicos y observaciones previas. Elabora } \\
\text { el plan de observaciones o experimentos y los argumenta } \\
\text { utilizando principios científicos y los objetivos planteados. } \\
\text { Realiza mediciones y comparaciones sistemáticas que } \\
\text { evidencian la acción de diversos tipos de variables. Analiza } \\
\text { tendencias y relaciones en los datos tomando en cuenta el } \\
\text { error y reproducibilidad, los interpreta con base en } \\
\text { conocimientos científicos y formula conclusiones, las } \\
\text { argumenta apoyándose en sus resultados e información } \\
\text { confiable. Evalúa la fiabilidad de los métodos y las } \\
\text { interpretaciones de los resultados de su indagación. }\end{array}$ \\
\hline Nivel 6 & $\begin{array}{l}\text { Nivel } \\
\text { esperado } \\
\text { al final del } \\
\text { ciclo VI }\end{array}$ & $\begin{array}{l}\text { Indaga a partir de preguntas e hipótesis que son verificables } \\
\text { de forma experimental o descriptiva con base en su } \\
\text { conocimiento científico para explicar las causas o describir el } \\
\text { fenómeno identificado. Diseña un plan de recojo de datos } \\
\text { con base en observaciones o experimentos. Colecta datos } \\
\text { que contribuyan a comprobar o refutar la hipótesis. Analiza } \\
\text { tendencias o relaciones en los datos, los interpreta tomando } \\
\text { en cuenta el error y reproducibilidad, los interpreta con base } \\
\text { en conocimientos científicos y formula conclusiones. Evalúa } \\
\text { si sus conclusiones responden a la pregunta de indagación y } \\
\text { las comunica. Evalúa la fiabilidad de los métodos y las } \\
\text { interpretaciones de los resultados de su indagación. }\end{array}$ \\
\hline
\end{tabular}




\begin{tabular}{|c|c|c|}
\hline Nivel 5 & $\begin{array}{l}\text { Nivel } \\
\text { esperado } \\
\text { al final del } \\
\text { ciclo V }\end{array}$ & $\begin{array}{l}\text { Indaga las causas o describe un objeto o fenómeno que } \\
\text { identifica para formular preguntas e hipótesis en las que } \\
\text { relaciona las variables que intervienen y que se pueden } \\
\text { observar. Propone estrategias para observar o generar una } \\
\text { situación controlada en la cual registra evidencias de cómo } \\
\text { una variable independiente afecta a otra dependiente. } \\
\text { Establece relaciones entre los datos, los interpreta y los } \\
\text { contrasta con información confiable. Evalúa y comunica sus } \\
\text { conclusiones y procedimientos. }\end{array}$ \\
\hline Nivel 4 & $\begin{array}{l}\text { Nivel } \\
\text { esperado } \\
\text { al final del } \\
\text { ciclo IV }\end{array}$ & $\begin{array}{l}\text { Indaga al establecer las causas de un hecho o fenómeno } \\
\text { para formular preguntas y posibles respuestas sobre estos } \\
\text { sobre la base de sus experiencias. Propone estrategias para } \\
\text { obtener información sobre el hecho o fenómeno y sus } \\
\text { posibles causas, registra datos, los analiza estableciendo } \\
\text { relaciones y evidencias de causalidad. Comunica en forma } \\
\text { oral, escrita o gráfica sus procedimientos, dificultades, } \\
\text { conclusiones y dudas. }\end{array}$ \\
\hline Nivel 3 & $\begin{array}{l}\text { Nivel } \\
\text { esperado } \\
\text { al final del } \\
\text { ciclo III }\end{array}$ & $\begin{array}{l}\text { Indaga al explorar objetos o fenómenos, al hacer preguntas, } \\
\text { proponer posibles respuestas y actividades para obtener } \\
\text { información sobre las características y relaciones que } \\
\text { establece sobre estos. Sigue un procedimiento para } \\
\text { observar, manipular, describir y comparar sus ensayos y los } \\
\text { utiliza para elaborar conclusiones. Expresa en forma oral, } \\
\text { escrita o gráfica lo realizado, aprendido y las dificultades de } \\
\text { su indagación. }\end{array}$ \\
\hline Nivel 2 & $\begin{array}{l}\text { Nivel } \\
\text { esperado } \\
\text { al final del } \\
\text { ciclo II }\end{array}$ & $\begin{array}{l}\text { Explora los objetos, el espacio y hechos que acontecen en } \\
\text { su entorno, hace preguntas con base en su curiosidad, } \\
\text { propone posibles respuestas, obtiene información al } \\
\text { observar, manipular, describir; compara aspectos del objeto } \\
\text { o fenómeno para comprobar la respuesta y expresa en } \\
\text { forma oral o gráfica lo que hizo y aprendió. }\end{array}$ \\
\hline Nivel 1 & $\begin{array}{l}\text { Nivel } \\
\text { esperado } \\
\text { al final del } \\
\text { ciclo I }\end{array}$ & $\begin{array}{l}\text { Explora los objetos, el espacio y hechos que acontecen en } \\
\text { su entorno, los observa y manipula con todos sus sentidos } \\
\text { para obtener información sobre sus características o usos, } \\
\text { experimenta y observa los efectos que sus acciones causan } \\
\text { sobre ellos. }\end{array}$ \\
\hline
\end{tabular}

Se puede observar cómo se requiere que el alumno evolucione de lo concreto a lo abstracto, sustentando su conocimiento en aquello que puede observar, logrando extraer conclusiones, hasta llegar al último nivel donde el alumno debe ser capaz de trabajar siguiendo el método científico.

En cuanto a los contenidos relativos a las ciencias en España van aumentado su dificultad de manera progresiva, partiendo desde conceptos más básicos hasta sexto con conceptos más complejos. Tanto la cantidad de contenidos cómo su complejidad va aumentando con la edad del alumno.

\section{Formación en ciencias de los docentes}

En cuanto a la formación del docente, en España nos podemos encontrar con que el único acceso para ser profesor de Educación Primaria, es cursando el grado de Magisterio en Educación Primaria, el cual tiene una duración de 4 años y 240 créditos, incluyendo prácticas en centros escolares. En cuanto a la formación en ciencias, se cursan 12 créditos concentrados en las asignaturas de 'didáctica del medio físico y químico' y 'didáctica del medio biológico y geológico'. 
Por otra parte, en Perú existe la posibilidad de realizar dicho grado, el cual tiene una duración de cinco años, pero también existen Institutos Pedagógicos de Educación Superior y Escuelas de Educación Superior de formación artística. La formación de profesores en estos Institutos y escuelas superiores se rigen por el diseño curricular nacional aprobado por el Ministerio de Educación. En cambio, cada facultad de Educación define su propio currículo con base en la autonomía que le otorga la ley a las universidades, y no siempre sigue los lineamientos de la política curricular y perfil de formación establecidos por el Ministerio de Educación. Por esta situación se tienen en la práctica varios perfiles, no siempre claros ni apuntando en la misma dirección. Asimismo, se carece de un sistema coherente y articulado de formación para los profesionales de la docencia. Si bien los estudios de la carrera en todas las instituciones de formación docente duran cinco años, quienes estudian en universidades tienen la ventaja de continuar su postgrado sin mayor dificultad. Por el contrario, los que egresan de un instituto superior pedagógico o de una escuela superior de formación artística deben realizar además un curso de complementación para obtener el bachillerato, condición para cursar una segunda especialización, maestría o doctorado. El postgrado es un requisito indispensable para ascender en las escalas superiores de la carrera pública magisterial (Díaz, 2015).

Una vez que poseen su titulación, por de estas vías, para trabajar para el Estado deben superar unas pruebas para lograr pasar de ser profesores contratados, a nombrados. Según la Ley de Reforma Magisterial en el capítulo V; el Ministerio de Educación autoriza, cada dos años, la convocatoria para el concurso público de ingreso a la Carrera Pública Magisterial, el cual se realiza en dos etapas: a) Primera etapa: está a cargo del Ministerio de Educación y evalúa las capacidades y conocimientos del postulante para el ejercicio de la docencia en la modalidad, forma, nivel y especialidad de las plazas en concurso. Se realiza a través de una prueba nacional clasificatoria. Consiste en la realización de un examen de matemáticas, de comunicación y otro de pedagogía, una vez superados estos exámenes, los cuales no median, sino que deberán estar todos aprobados para poder mediar, se puede realizar la segunda etapa. b) Segunda etapa, denominada de descentralización, está a cargo de la institución educativa, la cual ha elegido el futuro docente y evalúa la capacidad didáctica, formación, méritos y experiencia de quienes resulten aptos en la primera etapa. Esto lo evalúan por medio de una clase modelo que el futuro docente nombrado debe realizar, una entrevista y la entrega del currículum vitae.

En cuanto a la formación en ciencias de los futuros maestros, la formación solo se da en el curso denominado "Ecosistema". Para la formación complementaria del profesorado en ciencias, es una formación permanente a través de cursos impartidos en instituciones del Estado que cada docente debe pagar por cursar. Con estos cursos, solo se puede dar clases en el nivel en el que cada docente se encuentre. Esto se realiza para ampliar conocimientos sobre ciencias al final de obtener lo que equivaldría en España al grado universitario.

Según la web del Ministerio de Educación de Perú, en 2014, 95.668 docentes trabajaron sin título pedagógico de un total de 493.284 que laboran en la Educación Básica pública y privada en Perú: en Inicial son $27.4 \%$; en Primaria, $22.4 \%$, y en Secundaria, $12.2 \%$. En Lima, el $38 \%$ de los docentes no tiene título pedagógico y, en el sector privado, $66.3 \%$.

\section{Centros: contexto y enseñanza de las ciencias}

Con el objetivo de profundizar en los resultados obtenidos en la investigación inicial sobre varios aspectos relacionados con la enseñanza de las ciencias en ambos 
países, se llevaron a cabo estancias en un centro de Educación Primaria de Perú y en otro de España. En concreto, el centro educativo de Perú se trata del centro 10972 San Juan Masía, que se encuentra en el Caserío de Puplán, en Mórrope, localizado en la provincia de Lambayeque a 11,4 kilómetros de la ciudad de Chiclayo. El nivel socioeconómico de las familias es bajo, muchas de las familias viven de la agricultura de la zona ya que habitan en zonas próximas al centro, con lo que no poseen muchos recursos y una parte de las familias del centro son analfabetas con lo que no pueden ayudar mucho a los alumnos en su alfabetización científica.

En España, se ha trabajado en el Colegio Santa Ana, de Zaragoza. El barrio en el que se encuentra es eminentemente obrero, si bien se podría decir que es heterogéneo, tanto económica como culturalmente. Las principales ocupaciones son la industria, servicios y el sector de la construcción. El nivel socio-económico que domina la zona, y por lo tanto en las familias de nuestros alumnos, es de clase media, aunque detectamos que su nivel cultural es en muchas ocasiones medio-bajo. A los niños cuyos recursos son escasos y su economía deficiente, el centro les proporciona ayudas en la adquisición de materiales, uniformes y transportes o comidas. En la última década, el barrio ha acogido un gran número de familias inmigrantes $y$, por tanto, en el colegio ha aumentado el número de niños y niñas extranjeros/as, algunos de ellos con dificultades de idioma y de integración. En la actualidad cuenta con más de 900 alumnos con distintas realidades sociales, culturales y religiosas.

En ambos centros, la recogida de datos se llevó a cabo en clases similares de alumnos de la misma edad (7 años). A continuación, se muestran los resultados de la observación llevada a cabo durante dichas estancias.

A pesar de que el currículo oficial de Perú marca que en de $2^{\circ}$ de Primaria, se deben dedicar 3 horas/semana a trabajar ciencias y tecnología, la realidad es que en el centro tipo donde se han llevado a cabo las observaciones plasmadas en los resultados de este trabajo, eso no ocurría. En la práctica diaria no se cumplió con el horario. De manera sistemática, las clases diarias se dedicaron casi por completo a trabajar matemáticas y comunicación. Tras preguntar al maestro responsable de la clase el motivo, se concluye que éste considera más importante que los alumnos aprendan a leer, a escribir y a realizar cuentas básicas para poder desenvolverse en el día a día. Este alumnado debería saber leer y escribir, pero la realidad es que la mayoría no sabía. Por lo que queda patente que, en este centro no consideran importante las ciencias para el desarrollo del niño, se centran en la enseñanza de las matemáticas para tratar de desarrollar el razonamiento lógico y para que sean capaces de realizar cálculos con dinero en su vida cotidiana. También le dan mucha importancia a la enseñanza de la comunicación para, cómo dicen ellos que sepan 'firmar'. Dicha expresión significa: que sepan leer y escribir, para que nadie les engañe.

El centro educativo de Zaragoza contaba con un horario semanal, el cual se cumplía salvo que se realizaran salidas o actividades complementarias.

Otro aspecto recogido en las observaciones, es la elaboración de materiales didácticos propios. En España, partiendo de un contenido, son los propios docentes los que hacen las programaciones y unidades didácticas a trabajar, normalmente acorde al libro que el centro elige para los diferentes cursos. En Perú, es el estado el que les proporciona a los centros la editorial, los libros y los cuadernos que deben llevar (también disponibles online. En este caso, todo el material es gratuito para las familias, siendo el Estado el que asume su coste económico. Si el maestro prefiere utilizar otro material alternativo, puede hacerlo, siempre y cuando sean las familias las que asuman el coste. En Perú, es también el propio Estado el que programa los contenidos a impartir en cada una de las sesiones y el desarrollo de estas, especificando la 
duración de cada actividad, pero únicamente de las áreas de matemáticas, comunicación y educación física, no así de ciencias. Se pueden encontrar artículos que ayudan al desarrollo de la práctica docente en el contexto del centro peruano. Así, Gallego (2001) el habla de la literalidad en las comunidades andinas y rurales: 'Hay que tener en cuenta y comprender la actividad práctica como transformadora de la naturaleza social, para ello hay que estudiar el aprendizaje y participación de los niños indígenas en las tareas que realizan fuera de la escuela. Se debe intentar en todo momento que los alumnos sean capaces de elaborar ellos mismos sus aprendizajes con lo que pueden trabajar y ver en su día a día como el contacto con la chacra (campo) o con los diferentes animales a los que deben cuidar, ya que son el sustento de su familia'. En base a esta reflexión, para la explicación de las ciencias debería partirse de un conocimiento o concepto que los alumnos manejen en su día a día, de situaciones o fenómenos relacionados con el campo y el cultivo, o la ganadería, entre otros muchos temas y recursos que se podrían aprovechar, construyendo a partir de ahí su propio aprendizaje. Sin embargo, se observa que los docentes no salen del aula para aprovechar dichas situaciones favorables a la enseñanza de las ciencias a través de su entorno y no suelen utilizar los recursos que les rodean para partir de un hecho o acontecimiento de interés para el alumnado. Por otro lado, tampoco utilizan otras asignaturas como música o educación física para poder relacionar algún concepto y trabajar conocimientos de manera transversal.

Por otra parte, en el centro de España es muy utilizada la transversalidad en la enseñanza de los contenidos, es decir, utilizan asignaturas de artes, como plástica o música, para la enseñanza de otros conceptos más relevantes de ciencias. Por ejemplo, se ha observado que de manera habitual se utilizan las canciones para aprender diferentes temas de lingüística o incluso matemáticas.

El aula donde se ha llevado a cabo la observación en Zaragoza, está formada por 25 alumnos, la mayoría tienen un grado de desarrollo y las capacidades esperadas de acuerdo a su edad, además de tener unos conocimientos básicos coincidiendo con los habituales de su edad y su curso. Ninguno de ellos requiere de necesidades especiales de enseñanza, ya que su nivel lingüístico es adecuado y se comunican con frases estructuradas y vocabulario adecuado. En cuanto a la escritura y lectura, presentan pequeñas dificultades que se trabajan en el día a día. En general, tienen un ritmo de aprendizaje fluido y comprenden asimilando los conceptos con rapidez teniendo en cuenta su desarrollo cognitivo. Estos alumnos no tienen ningún problema en contestar a las preguntas que les hace la maestra, no tienen miedo al error. La relación entre los alumnos es afable a pesar de que al terminar la etapa de infantil ya no comparten clase con sus compañeros anteriores. La maestra-tutora le da mucha importancia a las relaciones entre ellos solucionando los conflictos que surgen de manera rápida y eficaz. Trabajan siempre en grupo colaborativo y cuando un alumno no entiende algo o no ha realizado alguna actividad, en primera instancia es otro compañero el que trata de ayudarlo.

En el aula de Perú, hay trece alumnos, de los cuales tres alumnas tienen dificultades de aprendizaje. Dichas alumnas no conocen el abecedario, con lo que no saben escribir palabras ni dictándoles las diferentes letras. El profesor atribuye la causa de esto a que la madre y el padre no tienen conocimientos básicos. Otros alumnos se encuentran en situación de repetir curso, pero sus familias no lo aceptan porque es faltar a su orgullo y deciden que no quieren que repitan, sino que les aprueben y pasen de curso, sin tener los contenidos mínimos para la promoción de curso. Por el contrario, hay otras alumnas, cuatro en concreto, que reciben enseñanza en casa 
porque sus madres son también maestras, y tienen mejor nivel académico, saben leer y escribir con soltura además de conocer todas las letras del abecedario y su uso. Se observa que los alumnos de este aula necesitan mucho apoyo tanto verbal como personal, por lo que de manera habitual las correcciones tienen carácter de refuerzo positivo.

En cuanto al profesorado de las aulas analizadas, el maestro de Perú construye sus sesiones partiendo de las del Estado y los libros que este le provee. Es decir, partiendo de las sesiones modelo dadas por el Estado. El maestro de España, es el encargado de crear sus propias sesiones partiendo del currículo, en cuanto a contenidos, criterios y competencias, diseñando ellos la programación anual con las correspondientes unidades didácticas y sesiones, para su posterior evaluación y calificación al alumnado. A esta misma conclusión llegaron García y Martínez (2001) cuando estudiaron el caso de un centro español: 'en cuanto a la procedencia de las actividades que no corresponden al texto habitual, más del $80 \%$ del profesorado que las realiza señaló la elaboración propia, tanto para ejercicios como para trabajo práctico, y algo menos de la mitad indicó que utilizaba otros libros de texto (el $46,7 \%$ y de $43,5 \%$ para cada uno de los tipos de actividades citados). El empleo de otros materiales (propuestas didácticas, actividades elaboradas en cursos de formación, libros divulgativos, etc.) fue citado por alrededor del $20 \%$ de los profesores."

\section{Discusión}

Es sabido que las ciencias se deben enseñar desde edades tempranas con ejemplos cercanos a la realidad del alumno y con materiales que sean útiles (Harlen, 2015). Conforme se va avanzando, en los diferentes cursos, debe aumentar la complejidad y la cantidad de conceptos a abordar (Brenneman, 2011). Según el estudio desarrollado sobre los currículos oficiales de Perú y España, esto se cumple en ambos, de manera que la legislación de los dos países marca que el alumno debe aprender ciencias desde los primeros años de escolarización y los contenidos científicos se van trabajando más profundamente conforme el alumno va avanzando en los distintos cursos académicos.

Para fomentar las vocaciones científicas de los alumnos, es muy importante la labor del docente. Para que esta labor sea eficaz y consiga motivar y despertar las inquietudes de los alumnos, es fundamental una buena formación del profesorado tanto en ciencias, como en didáctica. Tan importante como los conocimientos sobre dicha materia son las estrategias necesarias para llevar a cabo su enseñanza. Tener maestros bien formados en didáctica de las ciencias evita inseguridades a la hora de impartir las ciencias en la escuela. De acuerdo a los planes de estudio de los futuros maestros, tanto en Perú como en España se cursan pocas asignaturas relacionadas con las ciencias y la didáctica de éstas. Esto provoca en los maestros carencias tanto de contenidos, como de procedimientos a utilizar en el aula que dificultan el desarrollo científico del alumnado. Cuando un docente conoce el contenido a impartir y se siente cómodo con las estrategias didácticas a utilizar, es capaz de realizar actividades diferentes a las que propone el libro y crea su propio material, convirtiéndose en un buen recurso para el aprendizaje. Así, el maestro es capaz de partir de la práctica, y trabajar mediante la indagación, para que los alumnos investiguen y sean conscientes de su proceso de aprendizaje y así llegar a la comprensión de los aspectos teóricos. Además, un docente con una formación adecuada será capaz de llevar a cabo sesiones más dinámicas e interactivas, ya que no sentirán inseguridad y se verán capaces de dar respuestas a las preguntas que planteen los alumnos. 
En cuanto a la evolución de la educación, en ambos países se ha reducido la tasa de analfabetismo a lo largo de los últimos treinta años. Aunque en ninguno de los dos llega a ser del $100 \%$.

En base a los resultados recogidos en la observación en los centros, se puede concluir lo siguiente. A pesar de que la enseñanza de las ciencias está reglada en ambos países, en el centro educativo de Perú no se cumple, apenas se trabajan y las clases diarias se dedican mayoritariamente a trabajar las matemáticas y la comunicación. Las pocas ocasiones en las que se trabajan las ciencias, no se hace de manera interdisciplinar, sino por medio de la mera repetición y sin partir de elementos ni situaciones cotidianas. En el centro educativo de España, se trabaja más la experimentación, pero no siempre enfocada de la manera correcta y la indagación se practica poco.

En definitiva, se debería tener más en cuenta que la enseñanza de las ciencias mediante la indagación es un proceso necesario para que el alumno desarrolle su vocación científica desde los primeros años de escolarización. Para conseguir cada vez más vocaciones científicas entre los alumnos, se debería formar mejor a los maestros en la didáctica de las ciencias.

\section{Agradecimientos}

Los autores agradecen al proyecto MINECO EDU2016-76743-P su apoyo en el desarrollo de este trabajo, al grupo BEAGLE y al IUCA de la Universidad de Zaragoza.

\section{Referencias}

Brenneman, K. (2011). Assessment for preschool science learning and learning environments. Early Childhood Research \& Practice, 13(1), 1-9.

Cortés, A.L., de la Gándara, M., Calvo, J.M., Ibarra, J., Arlegui, J., Martínez, M.B. y Gil, M. J. (2012). Expectativas, necesidades y oportunidades de los maestros en formación ante la enseñanza de las ciencias en la Educación Primaria. Enseñanza de las Ciencias, 30, 155-176.

Cuevas, P., Lee, O., Hart, J. y Deakort, R. (2005). Improving science inquiry with elementary students of diverse backgrounds. Journal of Research in Science Teaching, 42, 337-357. http://dx.doi.org/10.1002/tea.20053

Díaz, H. (2015). Formación docente en el Perú, realidades y tendencias. Santillana. ISBN: 978-612-01-0221-3

Gallegos, C. (2001). El currículo de primaria basado en actividades como puente entre la cultura local y global: una experiencia educativa en la Amazonía Peruana. Cultura y Educación, 13:1, 73-92,

García, S. y Martínez, C. (2001). Qué actividades y qué procedimientos utiliza y valora en profesorado en Educación Primaria. Enseñanza de las Ciencias, 19(3), 433-452.

Harlen, W. (2015). Working with Big ideas of Science Education. Trieste (Italia): Science Education Programme of IAP.

Hewson, P.W. y Hewson, M.G. (1987). Science teachers' conceptions of teaching: implications for teacher's education. International Journal of Science Education, 9(4), 425-440.

Hodson, D. (1996). Practical work in school science: exploring some directions for change. International Journal of Science Education, 18(7), 755-760.
Izquierdo, M., Sanmartí, N. y Espinet, M. (1999). Fundamentación y diseño de las prácticas escolares de ciencias experimentales. Enseñanza de las Ciencias, 17(1). 45-59.

Liang, L.L. y Richardson, G.M. (2009). Enhancing prospective teachers' science teaching efficacy beliefs through scaffolded, student-directed inquiry. Journal of Elementary Science Education, 21, 51-66.

Nott, M. (1996). When the black box springs open: Practical work in school and the nature of science. International Journal of Science Education, 18(7), 807-818.

Oliva, J.M. y Acevedo, J.A. (2005). La enseñanza de las ciencias en primaria y secundaria hoy. Algunas propuestas de futuro. Revista Eureka sobre Enseñanza y Divulgación de las Ciencias, 2, 241-250.

Pujol, R.M. (2008). Pensar en la escuela primaria para pensar en la formación de su profesorado, desde la DCE, en el marco del nuevo grado. En M. R. Jiménez Liso (ed.). Ciencias para el mundo contemporáneo y formación del profesorado en Didáctica de las Ciencias Experimentales. Almería: Ed. Universidad de Almería, 354-361.

Vílchez, J.M., y Bravo, B. (2015). Percepción del profesorado de ciencias de educación primaria en formación acerca de las etapas y acciones necesarias para realizar una indagación escolar. Enseñanza de las Ciencias, 33(1), 185-202. 